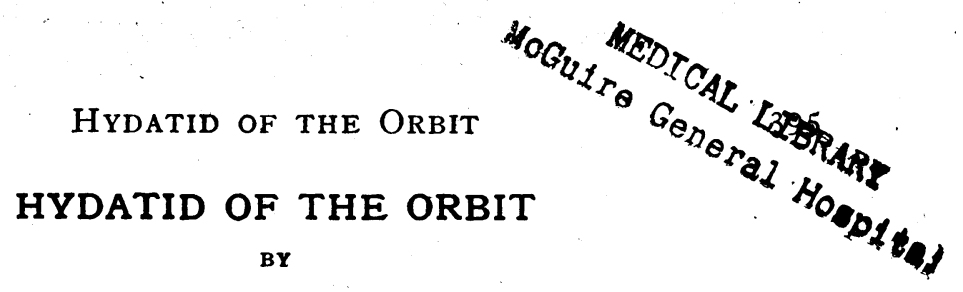

SiR HENKY HOLLAND, C.I.E.

INDIA

TEXT-BOOKs have very little to say regarding this condition. Fuchs merely mentions that cysts formed by the entozoa echinococcus and the cysticercus variety are found in the orbit. Duke-Elder states that these are not very rare in the orbit, although extremely rare intra-ocularly. Parsons contents himself with the statement that hydatid tumours are rare in England. Elliot's Tropical Ophthalmology treats the subject in some detail. He points out that it is common in the Argentine. Cabaut estimated that one in every 4,714 eye patients were cases of echinococcus of the orbit. This also refers to the Argentine. Demaria of Buenos Aires has also written on the subject in the Arch. d'Ophtal. on November, 1916, while Cabaut's paper was published on February 25, 1904. He wrote stating that though these cysts may develop in any part of the orbital cavity, they generally lie in or about the muscular cone, and he had never seen a case either within the optic nerve or within the eyeball.

My experience of this condition (eight cases) bears out Cabaut's findings. Those cysts that $I$ have seen are generally on the nasal side, and always in the upper fornix. I have also had the same experience as Demaria regarding the absence of any pericystic membrane in any of the cases. Seldom has pain been a predominant feature of this disease, and in some cases it has been entirely absent. The proptosis varies according to the duration of the condition. In the early stages there is only some fullness of the upper fornix while in the later stages the proptosis assumes such large proportions that the patient cannot close the eye, and there is often eversion of the lower lid, well marked chemosis, and occasionally ulceration of the cornea. Vision is in most cases excellent and $I$ have seen in two cases almost normal vision although the patient could not close the eye owing to the proptosis.

The cystic swelling requires to be differentiated from several other conditions, the most common being dermoid cyst and the varieties of exophthalmic ophthalmoplegia. The cystic swelling is for all practical purposes unilateral. The diagnosis may be clinched by aspirating the tumour and microscopic examination of the contents after the patient has been prepared for operation. If the typical hooklets are seen, the needle should be left in place and the cyst excised forthwith.

The treatment consists in removal of the cyst, entire if possible. This is an extremely difficult procedure owing to the friability of the 
cyst wall, and in these eight cases I have succeeded only once and my son, R. W. B. Holland has succeeded once. An incision is made into the conjunctival fornix nearest the tumour. The muscles are not usually divided, and blunt dissection enables the operator to display the bluish white cystic tumour. The contents are then aspirated, and with the needle still in place one half the volume of 20 per cent. formaldehyde is re-injected. An attempt is then made to remove the cyst entire with a pair of fixation forceps. If it tears, however, recourse must be made to a piecemeal removal. The cyst wall has often a very deep attachment, in which case it is difficult to be sure that the whole of the cyst wall has been removed.

The final result of the operation depends on whether the cyst has been removed in its entirety or not. Failing this, mild sepsis and a severe reactionary swelling and chemosis are apt to follow, even though the wound is drained with a fine rubberglove drain as a routine. The vision of the two patients, whose cysts were removed entire, was unimpaired. It is impossible to give an opinion of the ultimate visual result of the other six patients, because they did not report again to hospital after being discharged two or three weeks after the operation.

From this series of cases it is evident that the earlier the patient comes for treatment the better, since operative removal of a large cyst is rendered very much more difficult.

\section{A LOW POWER INFRA-RED MICROSCOPE}

BY

\section{T. Stuart-Black Kelly}

MANCHESTER

A DESCRIPTION has been given of the application of an infra-red viewing system to the penetration of corneal opacities ${ }^{1}$. The basis of the device is a German image converter tube that has the property of transforming an image in the infra-red region of the spectrum (at about 10,000 A.U.) into a visible image on a fluorescent screen. Through the courtesy of the Admiralty Research Laboratory we have been able to carry out a similar investigation using the equivalent British instrument. It has been confirmed that such equipment can be used with advantage when assessing the advisability of a corneal graft in certain cases.

Apparatus. The instrument in its present form was originally designed for an investigation of the variation of pupil diameter at very low levels of illumination. The viewing device, which can 